Check for updates

Cite this: Soft Matter, 2018, 14,4311

Received 11th January 2018, Accepted 6th April 2018

DOI: $10.1039 / \mathrm{c} 8 \mathrm{sm} 00091 \mathrm{c}$

rsc.li/soft-matter-journal

\section{Textured surfaces as a new platform for nanoparticle synthesis $\uparrow$}

\author{
Melih Baştopçu, $\ddagger^{\mathrm{a}}$ Anıl E. Derinöz $\ddagger^{\mathrm{b}}$ Asil C. Yılmaz ${ }^{\mathrm{b}}$ and E. Yegan Erdem (D) *bc
}

We present a new, surface-based microfluidic platform for the synthesis of nanoparticles. In this platform chemical reagents are carried in separate droplets, then mixed and later transported to a desired location on the surface using surface textured ratchet tracks. This brings the advantages of both synthesizing and transporting nanoparticles in situ without having cross-contamination between samples and addressing each sample independently. This platform is also capable of carrying multiple synthesis reactions concurrently.

\section{Introduction}

Microfluidic systems bring several advantages in nanomaterial synthesis such as precise control over reaction conditions (temperature, residence time, and concentration), small sample size, and rapid heating and cooling. In particular, droplet-based systems are very advantageous for nanoparticle synthesis as they eliminate cross contamination between samples, provide rapid mixing of reagents and allow uniform residence times for chemicals. ${ }^{1}$ Until now, nanoparticle synthesis via microfluidic methods has been carried out in microchannel based systems, where reagents were provided with syringe pumps and synthesis was held inside these microchannels. There are several examples of such systems in the literature. ${ }^{1-12}$

In channel based microfluidic systems, reagents are mixed inside channels, either in a droplet-based flow or in a continuous flow system, and nanoparticles are synthesized as a result of mixing and/or heating of these reagents. Compared to batch-wise methods, microfluidic techniques enable the synthesis of monodisperse nanoparticles. ${ }^{1}$ However, these systems require pumps, tubing, and valves which complicate the operation, and it is not possible to control the synthesis in a droplet by droplet fashion. In addition, synthesized nanoparticles may cause clogging in the microchannels. ${ }^{13}$

On the other hand, while providing all the advantages of microfluidic synthesis, surface based droplet microfluidics can

\footnotetext{
${ }^{a}$ Electrical and Electronic Engineering Department, Bilkent University, Ankara, Turkey

${ }^{b}$ Mechanical Engineering Department, Bilkent University, Ankara, Turkey. E-mail: yeganerdem@bilkent.edu.tr

${ }^{c}$ National Nanotechnology Research Center (UNAM), Ankara, Turkey

$\dagger$ Electronic supplementary information (ESI) available: Videos of nanoparticle generation on ratchet tracks. See DOI: 10.1039/c8sm00091c

\$ M. B. and A. E. D. contributed equally to this article.
}

eliminate the necessity of valves and tubing and therefore prevent leakage and clogging problems; they can address each reagent individually, and thus have very precise control over reaction. They have better programmability, and capability of carrying out multistep reactions where chemicals are introduced during the reaction. There are a few well known methods for droplet manipulation on surfaces and that are based on creating surface energy gradients such as electrowetting, thermocapillarity, chemical gradients and textured ratchets; ${ }^{13-22}$ and by using magnetic forces. ${ }^{23}$ Among these methods only electrowetting has been utilized for some chemical syntheses: Jebrail et al. demonstrated a combinatorial synthesis of peptidomimetics, ${ }^{13,17}$ and Dubois et al. synthesized tetrahydroquinolines. ${ }^{18}$ None of these surface based microfluidic manipulation methods have been utilized for nanoparticle synthesis yet, even though they provide all the necessary functions to perform such reactions.

The first demonstration of nanoparticle synthesis inside a droplet on a surface was shown by Abdelaziz et al. ${ }^{24}$ In this work gold nanoparticles were synthesized in a droplet by utilizing the Leidenfrost effect. However, in this method controllable droplet manipulation was not performed; the droplet in which the reaction took place was stationary.

In this paper we present the first surface based microfluidic droplet manipulation platform for synthesizing nanoparticles to address some of the limitations of channel-based microfluidic systems. In this platform droplets of reagents are transported on surface texture ratchets and we demonstrate the synthesis of magnetic iron-oxide and gold nanoparticles. Reagents are brought from separate tracks and later they are merged and mixed on a third ratchet track while being transported. This platform brings additional advantages such as individual and independent control of reagent droplets, simultaneous synthesis of different types of nanoparticles on the same platform and direct transport of nanoparticles on the surface after the synthesis 
without any clogging or cross-contamination problems. Therefore they are one of the most controllable platforms for the synthesis and manipulation of nanomaterials.

\section{Theory and design}

Surface texture can be used to direct droplets on surfaces by creating local surface energy gradients. ${ }^{19-21}$ Previously it was shown that texture ratchets can transport droplets in the "Fakir state" when a vertical vibration and asymmetric surface tension force is applied on the contact line of the droplet. ${ }^{19,20}$ In the Fakir state, droplets sit on the solid surface where, between the rough areas of the surface, air pockets are trapped underneath. This increases the hydrophobic properties of the surface, similar to rain droplets rolling on lotus leaves. ${ }^{25}$

Fig. 1 shows the schematic top view of a ratchet track. The tracks are composed of arc shaped rungs which provide asymmetry to maintain the non-uniform surface tension force on the advancing (front) and receding (back) edges of the droplet. When a droplet is placed on a track, its front contact line coincides with the whole arc, while the back contact line sits on a composite solid/gas interface. For a $3 \mu \mathrm{L}$ droplet sitting on such a track, the front contact angle is around $120^{\circ}$, while the back contact angle is $146^{\circ}$, which clearly shows the difference in wetting characteristics across the droplet. When the surface is vibrated vertically, the contact line of the droplet begins to oscillate and these oscillations turn into a net forward motion due to the higher pinning force at the front line. The advancing contact angle at the front edge of the contact line is higher than the advancing contact angle of the back edge, whereas the receding contact angle at the front edge is smaller than that at the back edge. This generates an asymmetric depinning force on the track which causes the droplet to move forward. ${ }^{19,20}$ Tracks are surrounded with arrays of pillars to provide superhydrophobic surroundings with a contact angle of up to $170^{\circ}$ to prevent droplets from leaving their route.

Ratchet tracks were previously used to transport water droplets; ${ }^{20}$ in this work they are modified to transport reagents of chemicals and later merge them on the surface for chemical

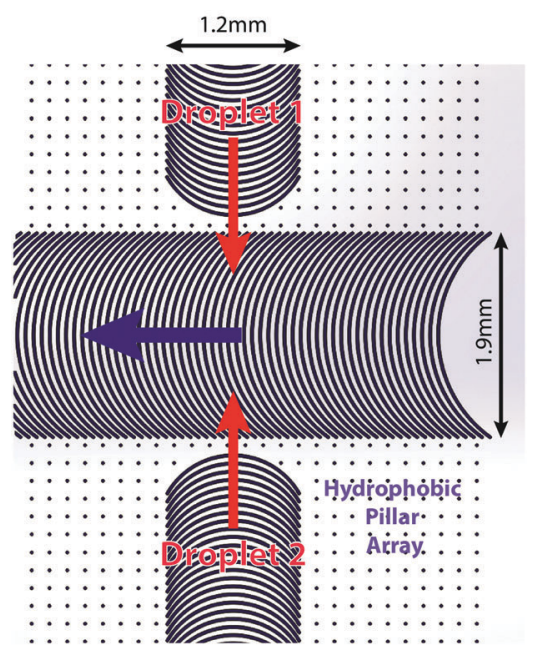

Fig. 1 Schematic top view of the ratchet tracks for nanomaterial synthesis. synthesis. The dimensions and design of tracks are different from those in ref. 20, though the mechanisms of transport are similar. Also this is the first demonstration of merging two tracks and their first application in nanomaterial synthesis.

For the synthesis of nanoparticles, we used two different track designs with different radii of curvature and widths to be able to transport smaller and larger volumes of droplets on the same surface. In both these designs, arcs had a width of $45 \mu \mathrm{m}$ with a gap of $30 \mu \mathrm{m}$ between them. Two tracks with a width of $1.2 \mathrm{~mm}$ and a radius of curvature of $0.7 \mathrm{~mm}$ merge into a larger track with a width of $1.9 \mathrm{~mm}$ and a radius of curvature of $1.06 \mathrm{~mm}$. The choice of the curvature is important as it should be suitable for the front contact line of the droplet to pin on it. For instance, for very large or much smaller curvatures the droplet will not be pinned at the front and therefore there will not be sufficient difference in the depinning forces at the front and back edges.

Droplets of reagents were carried on the side tracks, later they were merged on the larger middle track and transported to the desired area for collection or merging. During the transportation of the merged droplet, the reagents are mixed rapidly due to the oscillations and circulating flow inside the droplet. During this mixing, nanoparticles begin to be synthesized as will be discussed in the next section.

We have used two different synthesis platforms. In the first one, two tracks merge perpendicularly to each other to form a $\mathrm{T}$ junction where two reagent droplets meet and form a third droplet on the middle track. In this design the side tracks had a gap with the middle track and droplets merged on the middle track and two tracks did not have any shared geometry. This caused droplets to merge much more smoothly compared to designs that had intersecting side tracks with the middle track. We observed that the intersecting tracks at the T-junction caused pinning of the droplet permanently on the surface, and once they were merged, the resulting droplet did not move. The fabricated platform is shown in Fig. 2a.

In the other design, we used a platform which utilizes Y-shaped double junctions where four droplets of reagents can be manipulated to perform a concurrent synthesis of nanoparticles. The fabricated tracks are shown in Fig. 2b. Each of the Y-junctions has a different geometry to test different merging geometries. Both junctions were successful in merging droplets and transporting the third droplet on the middle track. The junction where side tracks intersected the middle track still worked unlike in the case of the T-junction since the tracks were oriented towards the direction of transport.

The driving frequency and amplitude of oscillations that are necessary for droplet transport depend on the volume and viscosity of the droplets since they move when they are excited at or near their resonance frequency. ${ }^{20}$ By selecting matching frequency-amplitude combinations, droplets with different volumes can be transported simultaneously, whereas at other combinations only droplets of corresponding volumes can be moved. Therefore, the frequency and amplitude ranges in which reagent droplets can be transported are mapped by keeping the acceleration constant while sweeping the frequency 


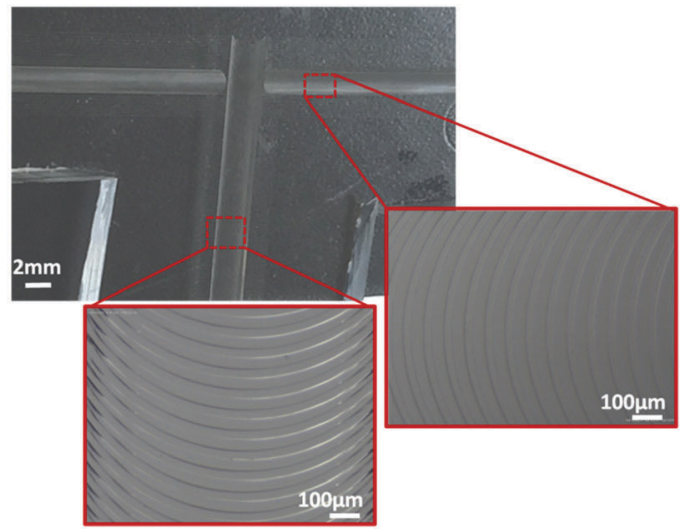

(a)

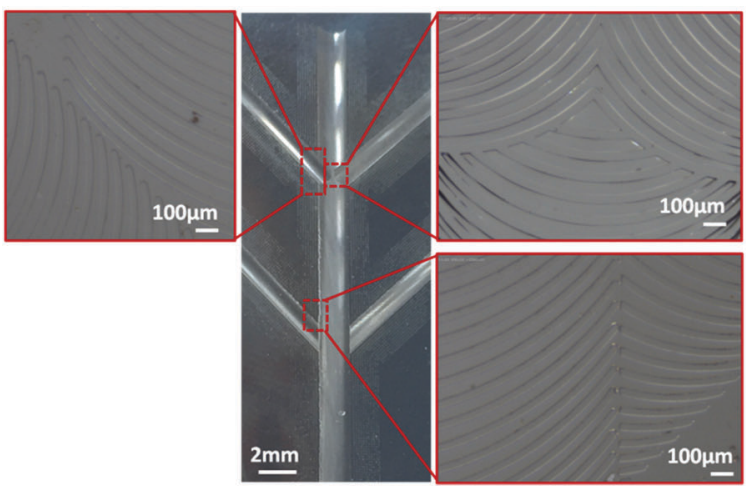

(b)

Fig. 2 (a) Ratchet tracks with a T-junction to synthesize nanoparticles. Snapshot images show the crossing region of the two tracks, the middle track and the side tracks. The tracks are surrounded by pillars to obtain a more hydrophobic area around them. (b) Ratchet tracks with double $\mathrm{Y}$-junctions. Each $\mathrm{Y}$-junction has a different geometry to try the possibility of merging and moving after merging for different designs. Both designs were successful in the transport and merging of droplets.

of oscillation. When vertical vibration is applied, the droplet begins to vibrate while its contact line is pinned on the surface. As the frequency is increased, its contact line also starts to oscillate. Afterwards the droplet can begin to move on the ratchet track. Even though the resonance frequency of the droplet is dependent on its surface tension, volume and viscosity, at different accelerations of oscillation, the frequency at which motion starts is different due to the parametric resonance behaviour of droplets. ${ }^{26}$ Therefore in order to find the range of frequencies at which the droplets can be transported, the lowest and highest frequencies at which there is motion are recorded. The mappings of the frequencies and amplitudes of reagent solvents used in the synthesis of iron-oxide and gold nanoparticles are shown in Fig. 3a-d. The reagents were a water based iron oxide solution and a base ( $\mathrm{NaOH}($ aq.)) solution for iron-oxide nanoparticle synthesis; gold chloride trihydrate and ascorbic acid with a sucrose solution for gold nanoparticle synthesis. Since the reagents were all aqueous, their maps were very close to each other. These data were collected for the side tracks where reagents were delivered. The ranges in which the sample can

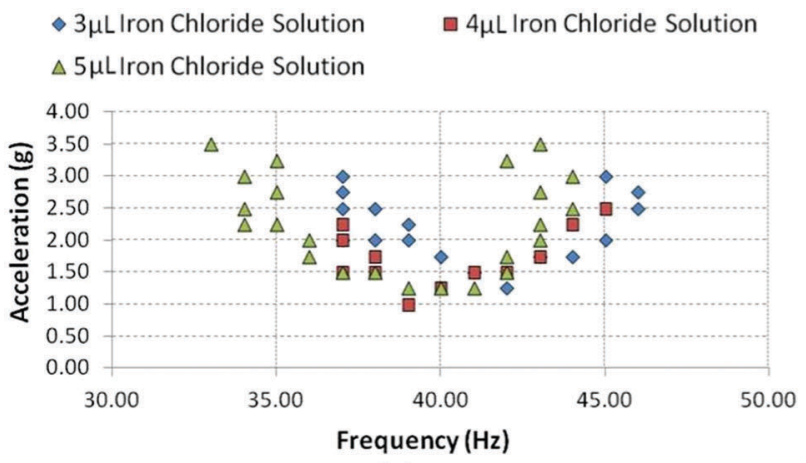

(a)

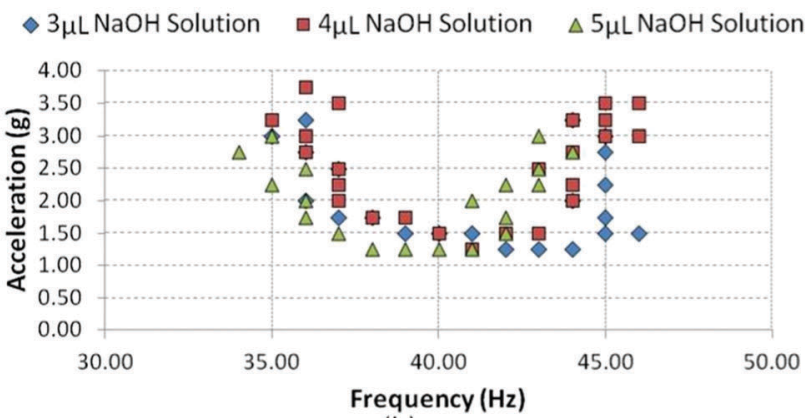

(b)

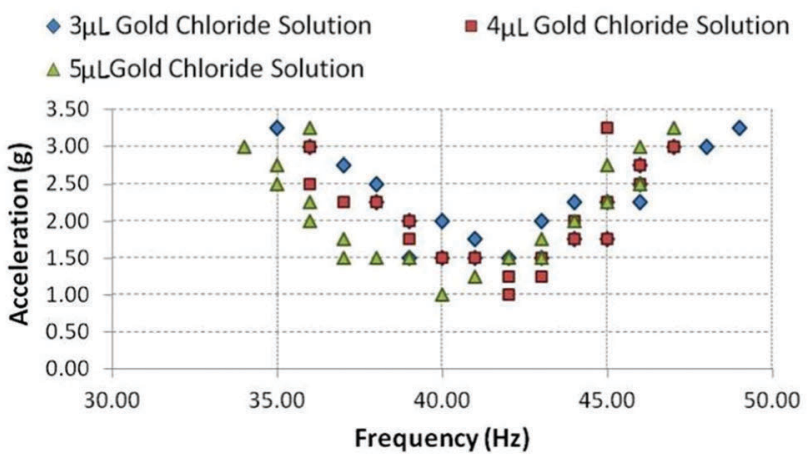

(c)

$\diamond 3 \mu \mathrm{L}$ Ascorbic Acid and Sucrose $\quad \square 4 \mu \mathrm{LAscorbic}$ Acid and Sucrose $\triangle 5 \mu \mathrm{L}$ Ascorbic Acid and Sucrose

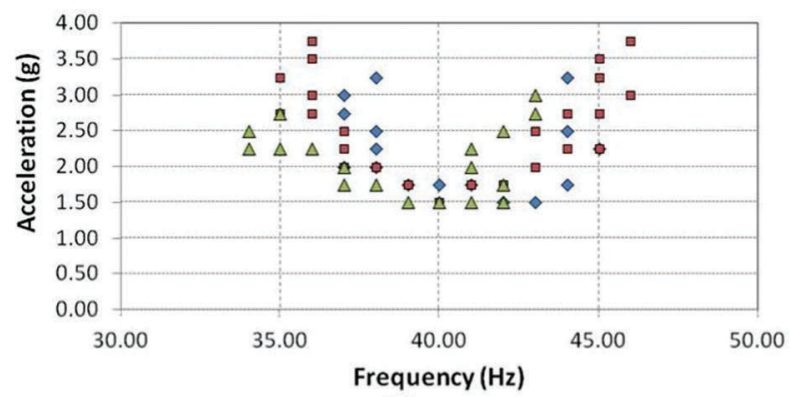

(d)

Fig. 3 Frequency and amplitude ranges in which the droplets of reagent solutions can be transported on the tracks. Data for the (a) aqueous iron chloride solution, (b) base solution, (c) gold chloride trihydrate solution, and (d) ascorbic acid and sucrose solution.

be moved on the tracks correspond to the area surrounded by the plots. Plots were taken for 3,4 , and $5 \mu \mathrm{L}$ droplet samples as these were the droplet ranges we used in the synthesis. The 
maximum droplet size that was transported on the ratchet tracks was $50 \mu \mathrm{L}$.

\section{Experimental}

Surface ratchet tracks were fabricated with polydimethylsiloxane (PDMS, Sylgard 184 Silicone Elastomer Kit) by soft lithography. A master mould of $70 \mu \mathrm{m}$ thickness was made out of a negative photoresist SU8-2050 (MicroChem Corporation) on a bare silicon wafer. Later PDMS was poured on the mould and cured at room temperature. Surface modification was not done on PDMS since its wetting characteristics were sufficient to keep aqueous droplets in the Fakir state.

For the synthesis of iron-oxide nanoparticles, iron(III) chloride hexahydrate (97\%, Sigma-Aldrich), iron(II) chloride tetrahydrate ( $\geq 99 \%$, Sigma-Aldrich), and sodium hydroxide (29\%, Fisher Scientific) were used to prepare aqueous solutions. The first reagent solution was composed of $0.075 \mathrm{M} \mathrm{FeCl}_{3}$ and $0.05 \mathrm{M}$ $\mathrm{FeCl}_{2}$. The second reagent solution was composed of $1 \mathrm{M}$ sodium hydroxide following the method reported by Karaağaç et al. ${ }^{27}$

For the synthesis of gold nanoparticles, two reagent solutions were prepared. The first one was $0.01 \mathrm{M}$ gold(III) chloride trihydrate solution, and the second one was a mixture of $0.05 \mathrm{M}$ ascorbic acid solution and $0.1 \mathrm{M}$ sucrose solution following the method reported by Hurtado et $a .^{28}$

After the synthesis, droplets containing the nanoparticles were collected inside glass tubes and cleaned several times with DI water using centrifuge and sonication. Later, they were observed under a transmission electron microscope (TEM).

The experimental set-up consisted of a function generator, a Brüel \& Kjaer Type 4809 vibration exciter, an oscilloscope, an accelerometer and a Phantom high-speed camera. All experiments were carried out at room temperature in air.

\section{Results and discussion}

The synthesis of iron-oxide nanoparticles was carried out by merging droplets of iron chloride and sodium hydroxide solutions with a 1 to 1 ratio at the junction of two ratchets. $3 \mu \mathrm{L}, 4 \mu \mathrm{L}$ and $5 \mu \mathrm{L}$ droplets were used for each reagent. When the reagents merge, they form a droplet on the third track. In order to transport the resulting droplet on the wider track, the frequency and amplitude of oscillations were changed. The corresponding ranges of frequencies and amplitudes used for experiments conducted with $3 \mu \mathrm{L}$ reagent droplets are shown in Fig. 4a. The resultant droplet moves on the third track that is wider than the side tracks; therefore, its mapping (green area) is slightly shifted compared to the other two reagent droplets. Another reason for this shift is that the resultant droplet has a larger volume and therefore its resonance frequency has changed. ${ }^{20}$

The synthesis of gold nanoparticles was also carried out by merging droplets of reagents with a 1 to 1 ratio at the junction of two ratchets. The corresponding frequency and amplitude ranges for experiments conducted with $4 \mu \mathrm{L}$ reagent droplets are shown in Fig. 4 b.

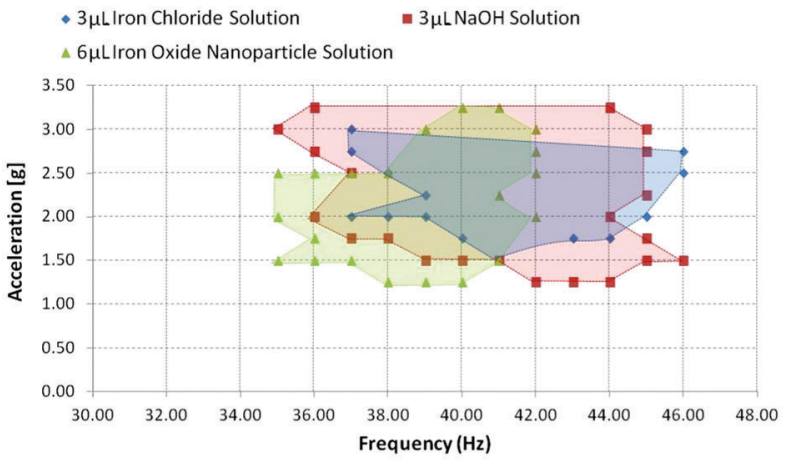

(a)

- Ascorbic Acid and Sucrose $(4 \mu \mathrm{L}) \otimes \mathrm{HAuCl} 4(4 \mu \mathrm{L}) \Delta$ Gold Nanoparticle Droplet $(8 \mu \mathrm{L})$

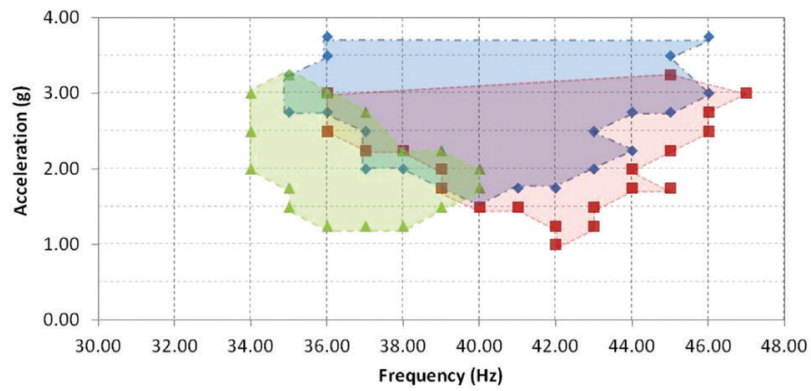

(b)

Fig. 4 Mappings of the reaction: (a) iron oxide nanoparticle synthesis mappings for reaction with $3 \mu \mathrm{L}$ of each reagent, and (b) gold nanoparticle mappings for reaction with $4 \mu \mathrm{L}$ of each reagent.

The snapshots during the synthesis of nanoparticles inside the droplets are shown in Fig. 5-7 for the T-junction with iron oxide nanoparticle synthesis and the Y-junction for gold nanoparticle synthesis and multiple synthesis on the same platform respectively.

It can be seen that as the synthesis reactions occur, the color of the merged droplet becomes darker due to the formation of

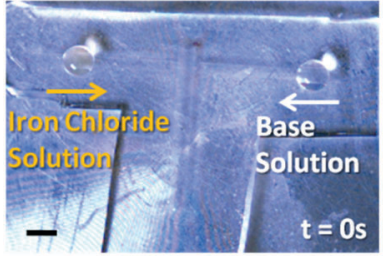

(a)

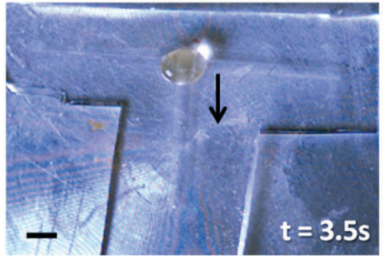

(c)

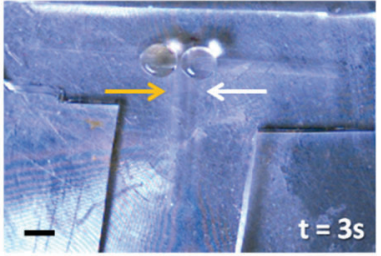

(b)

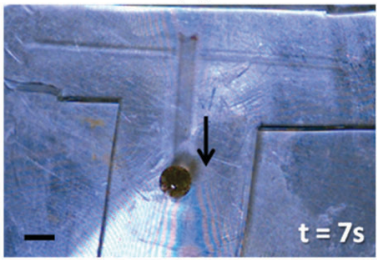

(d)
Fig. 5 Snapshot images of iron oxide nanoparticle synthesis on the surface ratchet tracks. Initially, the frequency and amplitude of oscillations are $40 \mathrm{~Hz}$ and $2 \mathrm{~g}$ ( $\mathrm{a}$ and $\mathrm{b}$ ). Once the droplets merge, the frequency and amplitude are readjusted to $32 \mathrm{~Hz}$ and $2.3 \mathrm{~g}$ (c and d) (scale bars: $2 \mathrm{~mm}$ ). 


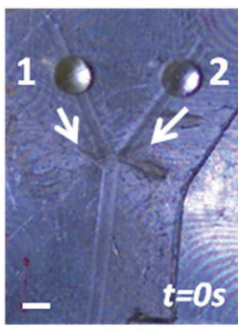

(a)

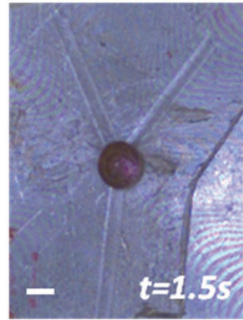

(c)

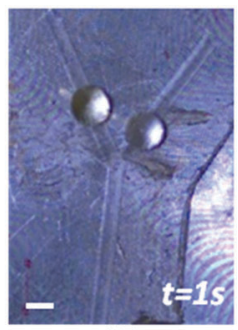

(b)

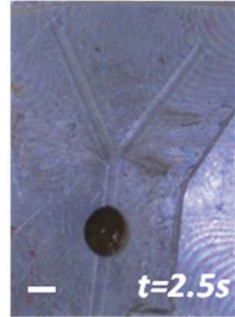

(d)
Fig. 6 Snapshot images of gold nanoparticle synthesis on the surface ratchet tracks with a $Y$ junction. Droplet 1 is gold chloride solution, while droplet 2 is ascorbic acid and sucrose solution. Initially, the frequency and amplitude of oscillations are $40 \mathrm{~Hz}$ and $2 \mathrm{~g}$ ( $\mathrm{a}$ and b). Once the droplets merge, the frequency and amplitude are readjusted to $35 \mathrm{~Hz}$ and $2.25 \mathrm{~g}$ (c and d) (scale bars: $2 \mathrm{~mm}$ ).

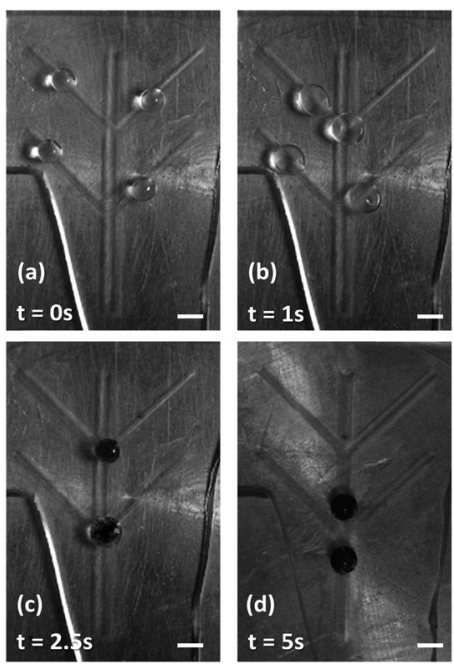

Fig. 7 Multiple synthesis on a double $Y$-junction device. Demonstration is done for iron oxide nanoparticles. The scale bar is $2 \mathrm{~mm}$.

nanoparticles. Also the droplet with nanoparticles was easily carried on the track without leaving any residue on the surface, which is essential for preventing material loss and contamination during the transport of nanoparticles.

For the gold nanoparticle synthesis, we have used a Y-junction. When the reagents merge at the junction, the amplitude and frequency of oscillations were shifted to the 'green' mapping area to be able to move the merged $8 \mu \mathrm{L}$ droplet.

It can be observed that in both gold and iron oxide syntheses, chloride solutions moved slower compared to the other reagent solution. The reason why droplets do not move at the same

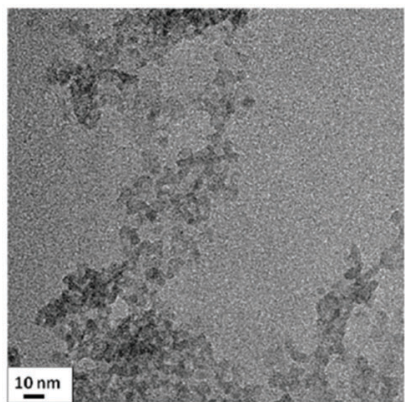

(a)

(c)

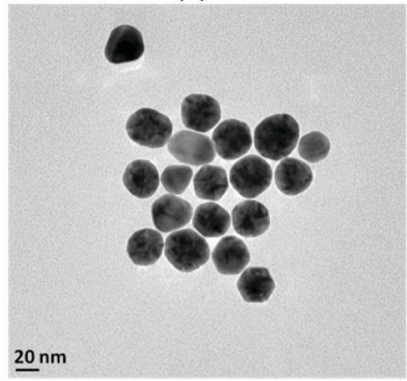

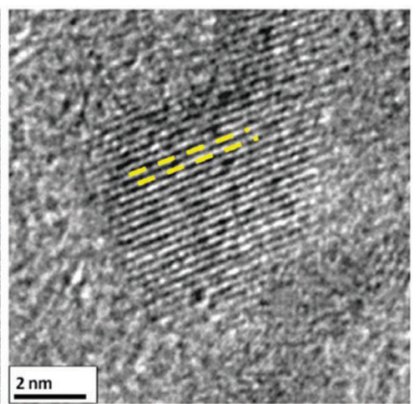

(b)

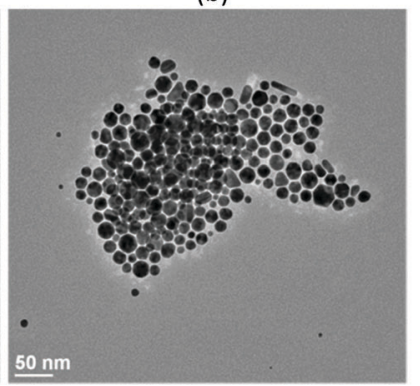

(d)
Fig. 8 ( $a$ and b) TEM images of synthesized iron oxide nanoparticles. The close up image on the right shows the crystal planes clearly (highlighted with yellow). The average sizes of the nanoparticles were $3.8 \pm 0.4 \mathrm{~nm}$, and the particles are more monodisperse compared to those obtained via batch techniques reported in the literature. ${ }^{27}$ ( $c$ and $d$ ) TEM images of synthesized gold nanoparticles. The average sizes of the nanoparticles were $16 \pm 3.8 \mathrm{~nm}$. Again the size distribution was better compared to those of batch techniques reported in ref. 28 .

speed is their difference in composition and therefore their surface tension, which changes their contact line oscillations on the track. ${ }^{20}$

After the synthesis and transport of the nanoparticles inside the droplets, samples were collected and observed under the TEM. As can be seen in Fig. 8a, iron-oxide nanoparticles were agglomerated; however, they can be still distinguished as individual, spherical shaped structures. In Fig. 8b, the crystal planes of particles can also be observed. These particles were magnetic and their sizes were $3.8 \pm 0.4 \mathrm{~nm}$. In Fig. $8 \mathrm{c}$ and d, TEM images of gold nanoparticles are shown. The size distributions of these particles were $16 \pm 3.8 \mathrm{~nm}$ and the particles were mostly spherical. The results can further be improved by adding ligands and increasing the track lengths so that the reaction time can be increased.

\section{Conclusion}

We have successfully demonstrated nanoparticle generation on a surface based microfluidic system composed of ratchet tracks. This technique was tested for both T-junction and Y-junction geometries. Each of the methods was successful in delivering reagents, mixing them on a third track and delivering the synthesized particles to the end of the track. A double Y-junction geometry can manage more than one synthesis at a time; it can also be used for the delivery of extra reagents during the 
synthesis, which opens up possibilities such as post-processing as well.

Compared to closed channel microfluidic techniques, this platform brings the advantage of addressing each of the reactants individually, preventing cross-contamination and clogging. On the other hand, extra reagents can be brought during the synthesis, multiple different types of nanoparticles can be synthesized in situ and the temperature can be externally controlled.

It is also possible to merge different volumes of droplets on these platforms. In order to do that, a frequency and amplitude combination at which both sizes of droplets can be transported needs to be selected.

Although the ratchet tracks were tested for a room temperature synthesis, they can also be used for higher temperature reactions if they are fabricated on substrates that can withstand higher temperatures. Also multiple tracks can be used in parallel to increase the throughput of the system. The introduction of reagents on the tracks can be automized using droplet dispensers.

\section{Conflicts of interest}

There are no conflicts to declare.

\section{Notes and references}

1 E. Y. Erdem, J. C. Cheng, F. M. Doyle and A. P. Pisano, Small, 2014, 10, 1076.

2 E. M. Chan, R. A. Mathies and A. P. Alivisatos, Nano Lett., 2003, 3, 199.

3 E. M. Chan, A. P. Alivisatos and R. A. Mathies, J. Am. Chem. Soc., 2005, 127, 13854.

4 A. J. deMello and A. deMello, Lab Chip, 2004, 4, 11N.

5 R. L. Hartman and K. F. Jensen, Lab Chip, 2009, 9, 2495.

6 S. Marre and K. F. Jensen, Chem. Soc. Rev., 2009, 39, 1183.

7 A. Abou-Hassan, O. Sandre and V. Cabuil, Angew. Chem., Int. Ed., 2010, 49, 6268.

8 A. M. Nightingale and J. C. deMello, J. Mater. Chem., 2010, 20, 8454.

9 A. M. Nightingale, S. H. Krishnadasan, D. Berhanu, X. Niu, C. Drury and R. McIntyre, Lab Chip, 2011, 11, 1221.
10 S.-K. Lee, X. Liu, V. S. Cabeza and K. F. Jensen, Lab Chip, 2012, 12, 4080.

11 S. Gomez-dePedro, C. S. Martinez-Cisneros, M. Puyol and J. Alonso-Chamarro, Lab Chip, 2012, 12, 1979.

12 A. M. Nightingale and J. C. deMello, Adv. Mater., 2013, 25, 1813.

13 M. J. Jebrail, N. Assem, J. M. Mudrik, M. D. Dryden, K. Lin, A. K. Yudin and A. R. Wheeler, J. Flow Chem., 2012, 2, 103.

14 A. A. Darhuber, J. P. Valentino, J. M. Davis, S. M. Troian and S. Wagner, Appl. Phys. Lett., 2003, 82, 657.

15 F. Mugele and J.-C. Baret, J. Phys.: Condens. Matter, 2005, 17, 705.

16 S. Daniel and M. K. Chaudhury, Langmuir, 2002, 18, 3404.

17 M. J. Jebrail, A. H. Ng, V. Rai, R. Hili, A. K. Yudin and A. R. Wheeler, Angew. Chem., Int. Ed., 2010, 49, 8625.

18 P. Dubois, G. Marchand, Y. Fouillet, J. Berthier, T. Douki, F. Hassine, S. Gmouh and M. Vaultier, Anal. Chem., 2006, 78, 4909.

19 A. Shastry, M. J. Case and K. F. Böhringer, Langmuir, 2006, 22, 6161.

20 T. A. Duncombe, E. Y. Erdem, A. Shastry, R. Baskaran and K. F. Böhringer, Adv. Mater., 2012, 24, 1545.

21 H. R. Holmes and K. F. Böhringer, Microsyst. Nanoeng., 2015, 1, 15022.

22 Y. Zhang and G. Wittstock, Small, 2017, 13, 1601691.

23 D. Tian, N. Zhang, Z. Zheng, G. Hou, Y. Tian, Y. Du, L. Jiang and S. X. Dou, ACS Nano, 2016, 10, 6220.

24 R. Abdelaziz, D. Disci-Zayed, M. K. Hedayati, J.-H. Pöhls, A. U. Zillohu, B. Erkartal, V. S. K. Chakravadhanula, V. Duppel, L. Kienle and M. Elbahri, Nat. Commun., 2013, 4, 2400.

25 P. G. De Gennes, F. Brochard-Wyart and D. Quéré, Capillary and Wetting Phenomena, Springer, New York, 2004, p. 221.

26 X. Noblin, A. Buguin and F. Brochard-Wyart, Phys. Rev. Lett., 2005, 94, 166102.

27 O. Karaağaç, H. Kockar, S. Beyaz and T. Tanrısever, IEEE Trans. Magn., 2010, 46, 3978.

28 R. Britto Hurtado, M. Cortez-Valadez, L. P. Ramírez-Rodríguez, E. Larios-Rodriguez, R. A. B. Alvarez, O. Rocha-Rocha, Y. Delgado-Beleño, C. E. Martinez-Nuñez, H. Arizpe-Chávez, A. R. Hernández-Martínez and M. Flores-Acosta, Phys. Lett. A, 2016, 380, 2658. 